\title{
Estado general de la investigación sobre videojuegos: un breve análisis y posibles tendencias a futuro
}

\section{General state of research on videogames: a brief analysis and possible future orientations}

Roy González-Sancho

Universidad Estatal a Distancia, Costa Rica

rgonzalezs@uned.ac.cr

\begin{abstract}
Resumen
Este artículo analiza el estado general de los estudios sobre los videojuegos al partir de varias revisiones sistemáticas de literatura y metaanálisis realizados en España y Latinoamérica. Entre los resultados se muestran los principales campos de estudio, desde los cuales se han tratado de comprender las ventajas y las desventajas de estos a saber: salud, educación y violencia. Asimismo, se muestra que existen faltas de rigurosidad, según las autoras y los autores, en las investigaciones relacionadas con los campos de la educación y la violencia, caracterizadas particularmente, por deficiencias teórico metodológicas, además de juicios de valor y estereotipos que no permiten comparar sus resultados o su validez. Ante esto, se concluye que, además de subsanar estas falencias, se hace necesario una profunda revisión de la reflexión al concepto, características o propiedades del juego de video, así como indagar otros aspectos como sus derroteros ideológicos-políticos, desde los cuales se producen.
\end{abstract}

Palabras claves: videojuegos, investigación social, salud, educación, violencia. 


\begin{abstract}
This article aims to analyze the state of the research on videogames from several systematic reviews of literature and meta-analysis, made mostly in Spain and Latin America. Among the results, it was found the main fields of study from which it has tried to understand the advantages and disadvantages of video games namely: health, education and violence. It also shows that there is a failure of rigor, according to the authors, in research related to the fields of education and violence, characterized mainly by theoretical, methodological deficiencies, as well as value judgments and stereotypes that produce an impossibility to compare or validating its results among papers. This article concludes that in addition to correcting these shortcomings, is necesary a deep research of the reflection on the concept, the characteristics or the properties of the videogame, as well as the others as the ideological-political courses from which they are produced.
\end{abstract}

Key Words: Video games, Social research, Health, Education, Violence.

\section{Introducción}

$\int$ a evolución de la industria del videojuego en términos de ganancias y los cambios que ha sufrido en los últimos 10 años ha sido sumamente acelerada. De acuerdo con Sánchez (2009), para el año 2008, este sector de la industria del entretenimiento recaudó en el nivel global un total de 32 mil millones de dólares -MdD-, monto que habría de seguir creciendo en los años siguientes, hasta cuadriplicarse en 2018, llegando a generar a 135 mil MdD aproximadamente, según los datos aportados por Batchelor (2018).

Ya desde aquel entonces, investigadores como Mäyrä (2008), conocían que el mercado de los videojuegos crecería de la mano con las mejoras en las conexiones a Internet, hacia una incorporación de nuevas plataformas -como los teléfonos móviles- ${ }^{-}$vías de acceso a los contenidos interactivos en línea, con una dinámica de comercialización de estos por parte de distribuidoras y desarrolladoras como un servicio, con miras a obtener de esta variable su mayor cantidad de ingresos ${ }^{1}$.

Sin embargo, no solo la industria ha venido desarrollándose e incluyendo cambios en su funcionamiento con el tiempo, sino también, las formas de abordaje y comprensión del videojuego. Como han señalado Wolf y Perron (2005), el estudio de estos ha progresado desde aquellos artículos publicados en revistas de tecnología e informática escritos por personas aficionadas y profesionales de esta disciplina, sobre los juegos de cómputo -así llamados en los 70-, a convertirse en un conjunto de corrientes de pensamiento, análisis e investigación con especialistas de varias

1 De hecho, para 2018, de acuerdo con Batchelor (2018), y Wijman (2018), el 51,85\% de los ingresos de la industria provinieron de lo recaudado por dispositivos móviles (microtransacciones, principalmente). 
áreas de las ciencias sociales, las artes, las ciencias de la salud y la educación.

Así, varios autores desde los años 90 comenzaron a llamar a este campo de investigación Game Studies o estudios del juego. De acuerdo con Hernández, Cano y Parra (2015), las indagaciones de este tipo son aquellas que se avocan a explicar cómo se compone y de qué trata el fenómeno del juego de video, agrupándose en tres paradigmas que los constituyen: constitutivos u ontológicos, metodológicos y de campo.

En el presente artículo se ha realizado un análisis de las principales tendencias de investigación sobre videojuegos en América Latina y España especialmente, con base en el estudio de una muestra seleccionada de Revisiones Sistemáticas de Literatura (RSL) y Metaanálisis publicados en diversas revistas académicas o de divulgación científica.

De esta forma, la gran parte de los documentos analizados en esta indagación, corresponden a estudios que podrían pertenecer a los paradigmas metodológicos y de indagaciones de campo. Pues, si bien, se encontraron una cantidad importante de trabajos individuales pertenecientes al paradigma constitutivo u ontológico, casi todas las RSL y metaanálisis han profundizado en estudios del segundo y tercer paradigma de los llamados Game Studies.

Explicitado lo anterior, se muestran los resultados de esta revisión del estado de la investigación sobre videojuegos de la siguiente manera: se detalla la metodología que fundamenta el trabajo realizado, seguido de los resultados obtenidos en la primera y la segunda etapa de análisis, la discusión de estos y las conclusiones del proceso, así como una breve exposición de las posibles vertientes de interés que podrían seguir futuros procesos de investigación.

\section{Metodología}

Como se ha dicho anteriormente, este trabajo ha tenido por objetivo principal dar cuenta de las principales tendencias de investigación que se han consolidado en el campo del estudio del videojuego, al partir del análisis de diversas publicaciones realizadas a través de Revisiones Sistemáticas de Literatura o Metaanálisis, por varias personas académicas y profesionales de diversas disciplinas.

Para ello, se utilizó de un enfoque cualitativo, con un alcance descriptivo-analítico, y se desarrolló en formato de ensayo científico. El cual, siguiendo los aportes de Guanipa (2008), y Rodríguez (2007), es una redacción sistemática y fundamentada en resultados de estudios propios o de terceros, que una persona investigadora realiza, al partir de su reflexión o posición subjetiva con respeto a un área específica del saber, que tiene como atribución central profundizar en ello, y así aproximarse a la realidad o las dinámicas propias del objeto, fenómeno o evento a tratar.

El análisis aquí presentado parte de productos académicos basados en RSL o metaanálisis, debido a la importancia que este tipo de trabajos ha ganado recientemente, al ser en la mayoría de los casos el resultado de un esfuerzo por analizar grandes cantidades de información, datos 
y adelantos en los diferentes campos del conocimiento, y que han llevado un proceso de refinamiento, significativamente riguroso, de las fuentes primarias analizadas. Por otra parte, este tipo de publicaciones ofrece una mejor radiografía de las grandes tendencias de investigación y producción académica existente y sus resultados.

De esta forma, se realizó una búsqueda de artículos o publicaciones de RSL y Metaanálisis en las siguientes plataformas electrónicas de acceso abierto: Red de Revistas Científicas de América Latina y el Caribe, España y Portugal (Redalyc.org), Google Académico (Scholargoogle.com) y la Scientific Electronic Library Online (Scielo.org) ${ }^{2}$. Para llevar a cabo la búsqueda de los artículos se utilizaron los descriptores: videojuegos, revisión sistemática, metaanálisis, game studies, investigación, juego y revisión.

Así, en una primera fase de revisión se encontraron un total de 93 productos académicos. Sin embargo, se procedió a realizar un filtro para descartar aquellos estudios que no tenían que ver con juegos de video, que no aportaban información relevante sobre las tendencias de investigación, o que no fuesen una comunicación o un producto -artículo- resultado primordialmente de una RSL o un Metaanálisis. Finalmente, la cantidad de escritos por analizar y que cumplía con las tres condiciones mencionadas fue de 37 , en total.

2 La escogencia de estas tres plataformas se debió principalmente a que arrojaron la mayor cantidad de resultados, en comparación de otras de acceso privado que no dieron resultados satisfactorios con los descriptores usados para este estudio.
El procesamiento y el análisis de los artículos se realizó con el programa informático Atlas.ti 7, el cual de acuerdo con Friese (2013), permite analizar datos cualitativos de texto, imágenes, audio y video, sin estructurar, para los cuales el uso de métodos estadísticos no es una opción adecuada en función de los objetivos de la investigación.

A partir, de las acciones que posibilita el programa, se creó una Unidad Hermenéutica (o archivo de trabajo) con los textos seleccionados. Luego se realizó una lectura preliminar de estos, con la cual se obtuvo como resultado una serie de códigos que se agruparon en tres familias (o conjuntos) por su afinidad, las cuales fueron finalmente: educación, salud, violencia y población. Acto seguido se realizó una lectura de todos los documentos asignándose a cada una de las diferentes citas seleccionadas en una de las diferentes categorías mencionadas, fue esta la fase de análisis básico.

Una vez realizado este procedimiento, se prosiguió con la siguiente etapa de análisis, de nivel conceptual, para esta el programa informático permite observar una tabla de coocurrencias o superposición de códigos asignados en las citas textuales seleccionadas, en la etapa anterior, mostrando las intensidades de coincidencias que estos tienen entre sí. De la misma forma, el Atlas.ti 7 permitió llevar un registro de comentarios y análisis - memos-, los cuales fueron importantes a la hora de la redacción de los resultados, la discusión y las conclusiones de este ensayo. 
Esta estrategia de análisis basada en dicha aplicación informática posibilitó que en este trabajo pudieran dibujarse los matices de las tendencias de investigación predominantes, manteniendo la coherencia interna de los resultados aquí presentados.

\section{Resultados}

\section{Primera fase de análisis, datos generales de los documentos de partida}

En este primer acercamiento, de los 37 documentos analizados se puede mencionar que 18 son publicados en España, 4 en México, 2 en Argentina, 2 en Colombia, 2 en Chile, 2 en Ecuador, 2 en Inglaterra y con una publicación se encuentran Costa Rica, Portugal, Perú, Estados Unidos y Brasil. Es decir, un $41 \%$ son de países latinoamericanos, un $48 \%$ de España, el restante 11 $\%$ fueron de países anglosajones.

Así mismo, 4 de estos son tesis de grado, 4 noticias sobre metaanálisis o una RSL, 1 un capítulo de un libro, y los restantes 28 son artículos publicados en revistas académicas. En cuanto a los años de publicación se tiene que 1 es de 2019, 9 son del año 2018, 7 del año 2017, 4 del año 2016, 3 de 2015, 3 de 2014 y las restantes 16 son de 2013 a 2005 la más antigua.

Por otra parte, al analizar de forma general los temas de interés de las publicaciones mediante la codificación realizada para este trabajo, se pudo observar que 19 se interesaron por asuntos relativos al estado de la investigación, en cuanto a videojuegos y salud, 12 se enfocaron con aspectos conectados con su uso y procesos de educación formal, 4 estudiaron a fondo la relación de estos con la violencia. Mientras que 2 artículos abordaron el desarrollo de las tendencias generales de estudio sobre estos, sin embargo, con abordajes diferentes al propuesto en este trabajo.

Finalmente, en cuanto a las poblaciones más estudiadas se encontró que la infantil, ha sido mayormente objeto de aproximaciones en este tema, particularmente, en relación con los usos de juegos de video en educación -fructíferos o mal obrados-, los efectos psicológicos de estos en los menores, y las alternativas exploradas en cuanto a su aplicación en el campo educativo formal, como recurso didáctico y los posibles beneficios que pueden tener. Por parte de las investigaciones relacionadas con jóvenes, la investigación parece haberse mostrado centrada en el uso de videojuegos en el centro educativo y sus beneficios, así como en los efectos físicos del uso de estos.

\section{Segunda fase de análisis, estudio conceptual}

Concordando con algunos autores y autoras de las pesquisas analizadas para este artículo (Planells, 2013; Ramón, 2018), dependiendo de las posturas tomadas, podría tornarse pretensioso hablar específicamente de un campo de estudio del juego de video formalmente constituido. Sin embargo, la considerable cantidad de investigación multidisciplinaria en España y Latinoamérica, realizada sobre el videojuego en sus diferentes facetas, usos, posibilidades, ventajas e implicaciones hace que un esfuerzo por reflexionar sobre sus vertientes de investigación no sea para nada poca cosa. 
En este sentido, como se mencionó anteriormente, se lograron identificar tres campos desde los cuales las investigaciones se han acercado al estudio del videojuego, a saber: salud, educación y violencia.

\section{Salud y juegos de video: ventajas y beneficios}

Alrededor de la salud, o más bien de la pregunta de ¿qué tan saludable es usar juegos de video?, las investigadoras y los investigadores del tema se han preguntado por las ventajas o los beneficios que tiene el uso de estos sobre varias áreas de la salud.

Aparece de esta manera, una primera aplicación con pacientes hospitalizados bajo procesos de neurorrehabilitación, básicamente con el objetivo de brindar tratamientos con metodologías alternativas para el "entrenamiento cerebral" de personas con alguna discapacidad o adultas mayores destacándose el caso de una aplicación llamada Luminosity, entre otras de las que hacen falta registros o investigaciones sobre sus efectos.(Gómez del Castillo, 2005; Gray, 2014; Merino y Vásquez, 2016; Mercola, 2017; Vela, Velázquez y Veyna, 2017; Stanmore, Stubbs, Vancampfort, de Bruin, y Firth, 2017; Parra-Moreno, Rodríguez-Juan, y Ruiz-Cárdenas, 2018; Sánchez, Collado, Martín, y Cano, 2018; Escaravajal y Martín-Acosta, 2019)

Además, el uso de juegos de video ha probado ser una herramienta útil en procesos de rehabilitación, tanto de la mano con la aplicación de interfaces cerebrocomputadora, como complementando los tratamientos habituales en este campo. De acuerdo con las personas autoras, los beneficios se han visto en los abordajes de enfermedades relacionadas al sistema motor como distrofia muscular, lesiones de la médula espinal, esclerosis, parálisis cerebral, entre otras. Esto debido a que se trabajan o reentrenan habilidades motrices y reflejos, teniendo potenciales preventivos frente al deterioro de estas, especialmente, si se habla de personas de la tercera edad. (Gómez del Castillo, 2005; Vela et al., 2017; Ortiz-Huerta, Pérez-de-Heredia-Torres, Guijo-Blanco, y SantamaríaVázquez, 2018; Sánchez et al., 2018; Parra-Moreno et al., 2018)

En el nivel cognitivo, dentro de los beneficios del uso de juegos de video activos y del tipo habituales -arcade, rol, rompecabezas y plataformas- se encuentran en el mejoramiento de áreas de control, memoria de trabajo y atención (general, selectiva, alternante), las habilidades visoespaciales (es decir de manipulación de objetos de forma mental), de percepción y navegación, entre otras funciones ejecutivas. Las cuales tienen un papel importante en la mejora de las destrezas para la resolución de problemas y desarrollo intelectual. (Pindado, 2005; Tejeiro, Pelegrina del Río, y Gómez, 2009; Beltrán-Carrillo, Valencia-Peris, y Molina-Alventosa, 2011; Moncada y Chacón, 2012; Gray, 2014; Toril, 2015; Stanmore et al., 2017; Mercola, 2017; de Oliveira, Mena, y de Lima, 2018)

En un sentido similar algunas investigaciones han mencionado que, con el uso de juegos de video en poblaciones de personas adultas y de la tercera edad sanas, ha sido posible detectar muestras de plasticidad cerebral en estas, además de poder observar que su uso posibilita mejorar 
además de las habilidades señaladas anteriormente, la memoria episódica y de trabajo. (Soto, 2014; Toril, 2015; Merino y Cárcamo, 2016)

Por otra parte, los beneficios también se han traducido en ciertas ganancias motivacionales, pues tanto en los ensayos clínicos registrados como en las terapias en las que el uso de videojuegos es un eje central, los resultados comunicados por las personas autoras detallan que la adherencia y la satisfacción de las personas con respecto a los procesos terapéuticos es mayor, además de ser una herramienta de bajo coste en comparación con otras disponibles en el mercado de la salud, tanto en entornos hospitalarios como domiciliarios de América Latina y España. Al mismo tiempo, se ha mencionado que de los videojuegos activos tienen el potencial de convertirse en una posible forma de entrenamiento en hábitos saludables, comportamientos prosociales (aunque hace falta más investigación) y de combate a epidemias como el sedentarismo o la obesidad. (Tejeiro et al., 2009; Segalás, 2011; Beltrán-Carrillo et al., 2011; Etxeberria, 2011; Moncada y Chacón, 2012; Gray, 2014; Toril, 2015; Merino y Cárcamo, 2016; Humanante-Ramos, García-Peñalvo y Conde-González, 2017; Stanmore et al., 2017; Pascuas, Vargas v Muñoz, 2017; Ortiz-Huerta, et al., 2018; Parra-Moreno et al., 2018)

No obstante lo anterior, cabe tomar en consideración la advertencia realizada por Vela et al. (2017), quienes han señalado que una parte de los estudios han desarrollado sus procesos de experimentación, casi exclusivamente con personas sanas, lo cual no garantiza ciertamente, que los resultados vayan a ser los mismos con personas afectadas por una discapacidad, enfermedad o padecimiento.

\section{Desventajas o inconvenientes en salud por el uso de videojuegos}

Dentro de las mismas pesquisas referidas por las autoras y los autores se ha señalado la presencia de inconvenientes o desventajas que se asocian con el uso de videojuegos. Entre estos, en cuanto a la temática salud, el desarrollo de adicciones es uno de los más mencionados.

En principio, existen investigaciones en contextos hispanoamericanos y anglosajones que sostienen que el uso de cierto tipo de juegos de video podría alterar en el cerebro las áreas de procesamiento de recompensas, dando como el resultado el desarrollo de una adicción. Sin embargo, también se hacen llamados cautelosos al respecto, pues aunque es posible que las personas adquieran sujeciones patológicas por un objeto o actividad, no puede hablarse de forma mecánica que se trate de una adicción, sin preguntarse por las condiciones o las circunstancias que han llevado a esas personas a esa sujeción, especialmente, si se considera que en la actualidad (hasta la publicación de este artículo) no se cuenta con ningún dato que permita hablar de causalidad directa de adicción por usar específicamente, videojuegos. (Tejeiro et al., 2009; Lancheros, Amaya y Barquero, 2014; Valcárcel, 2013; Soto, 2014)

Asimismo, parece tenerse alguna certidumbre con respecto al riesgo que corren las personas con fotosensibilidad de sufrir ataques epilépticos por los destellos de 
luz rápidos o imágenes parpadeantes de algunos videojuegos y otros contenidos audiovisuales. Tal es el grado que varios fabricantes han recomendado proteger los ojos de la luz azul de las pantallas de televisores, celulares, etc., mediante filtros externos o funciones de apagado de los diodos de los pixeles de las pantallas mediante software de los dispositivos móviles, consolas o los mismos contenidos del juego. (Tejeiro et al., 2009; Valcárcel, 2013; Mercola, 2017)

Entre otras consecuencias físicas, personas autoras de América Latina, España y de países anglosajones han mencionado que se han reportado dolor de muñecas por movimientos repetitivos, desarrollo de estilos de vida sedentarios y obesidad, fatiga visual y mental, trastornos psiquiátricos y del sistema nervioso, problemas familiares, aislamiento social, etc. (Gray, 2014; Merino y Cárcamo, 2016; Ortiz-Huerta, et al., 2018; Uchuypoma , 2017).

En este apartado, también se han realizado señalamientos sobre algunos de los resultados presentados en las investigaciones revisadas, destacando que con frecuencia las polémicas que rodean a los juegos de video, se fundamentan en la mayoría de las ocasiones en prejuicios, sesgos, opiniones o argumentos morales que parten más de suposiciones que de evidencias comprobadas. Por otro lado, se ha apreciado que en varias ocasiones los estudios que argumentan los efectos nocivos de los juegos de video cuentan con modelos teóricos o metodológicos sin suficiente fundamentación, por lo que instrumentos, mediciones, conceptos, diagnósticos e interpretaciones aportadas, terminan siendo confusas o inexactas, dificultando llegar a datos o resultados concluyentes (Tejeiro et al., 2009; Lancheros et al., 2014; Soto, 2014).

\section{Videojuegos y educación}

Sobre este tema en particular, las inquietudes de las personas investigadoras en América Latina parecen haber nacido a partir de preguntarse cómo aprovechar o aplicar los conocimientos sobre las ventajas del juego tradicional mediante la incorporación de estos programas informáticos al trabajo docente y de aula en los entornos educativos formales o institucionales.

En este sentido, siguiendo a Vela et al. (2017), quizás una de las razones por las que se ha intentado incluir al videojuego en los ámbitos de educación formal ha sido por el papel que la actividad del juego tiene sobre la estructuración del lenguaje, el pensamiento y el desarrollo de aprendizajes significativos. Al tiempo que disminuye el miedo frente a los fracasos y los errores, permitiendo la participación individual o colectiva de las personas involucradas en las dinámicas lúdicas desplegadas y desarrollando la creatividad, el intelecto y la fortaleza emocional, entre otras propiedades.

\section{Beneficios aplicaciones de videojuegos y educación}

De acuerdo con las apreciaciones de las personas autoras la incorporación de juegos de video en entornos educativos latinoamericanos y españoles ha demostrado ser efectiva, esencialmente, en aquellas iniciativas en las que se explota 
su potencial interactivo y competitivo, así como la sensación de autonomía que experimenta la persona implicada. Ello debido a que estos parecen incidir en las estructuras cognitivas y procesos de pensamiento, generando en la buena medida aprendizajes y destrezas de un modo "rápido" y "efectivo" (Guerra, 2015; Oceja y González, 2018).

En este sentido, conociendo los beneficios que tienen los juegos de video en el área motora, cognitiva, emocional y perceptual de las personas de varios rangos de edad, se ha considerado que estos pueden aprovecharse como herramienta educativa siempre y cuando se utilicen desde un enfoque de aprendizaje y trabajo colaborativo, aunque también, se advierte la necesidad de aumentar la formación del personal docente en este aspecto, con el fin de facilitar los procesos de enseñanza (Pindado, 2005; Tejeiro et al., 2009; Moncada y Chacón, 2012; Martín del Pozo, García-Valcarcel y Basilotta, 2017).

Junto con las propiedades generales recién descritas, varias personas del ámbito de la investigación han recogido experiencias de estudios en las cuales se han aplicado videojuegos como parte de las herramientas de trabajo dentro de las aulas en contextos suramericanos y españoles, en particular aquellos, cuyas mecánicas y contenidos se desarrollaron principalmente, con el propósito de ser usados en las clases (Pindado, 2005; Marín, Alor, Zatarain y Barron, 2016; Merino y Cárcamo, 2016).

Dichos videojuegos diseñados con propósitos distintos al entretenimiento, han recibido el nombre de juegos serios o
Serious Games, y se caracterizan por centrarse en simulaciones, recreaciones, rompecabezas, etc., que tienen por objeto servir como medios o herramientas para promover ciertos valores, mensajes, habilidades, toma de decisiones, resolución de problemas, motivaciones con fines didácticos e informativos ${ }^{3}$ (Gómez del Castillo, 2005; Etxeberria, 2008; Valcárcel, 2013; Lozada y Betancur, 2016; Martín del Pozo et al., 2017).

Al mismo tiempo, este tipo de videojuegos son relevantes en el plano educativo debido a la capacidad de equilibrar el entretenimiento audiovisual y la jugabilidad con los objetivos de aprendizaje para los que sean utilizado (Etxeberria, 2008; Marín et al., 2016).

Asimismo, sobre la aplicación de estos juegos de video en entornos educativos formales, se han destacado acotaciones sobre las motivaciones que brinda a las personas que juegan en el área cognitiva, tales como la facilidad de incentivar el uso de razonamiento lógico y el pensamiento inductivo, asimismo han sido señaladas ciertas mejoras en el rendimiento de habilidades motoras esencialmente, en aquellas poblaciones en edad escolar. En fin, la posibilidad de potenciar habilidades de pensamiento o conocimientos de forma diferenciada y lúdica, ha permitido resultados sobresalientes en relación con los estilos tradicionales de enseñanza (Pindado, 2005; Etxeberria, 2008; Guerra, 2015; Merino y Cárcamo, 2016).

3 En esta misma categoría, también pueden ser incluidos aquellos juegos de video utilizados o diseñados para el área de la salud y la rehabilitación de personas con padecimientos motor o neurológico. 
Por otro lado, en el contexto español se ha venido consolidando la aplicación de las mecánicas y las técnicas de diseño de juego a entornos tradicionalmente no lúdicos como empresas o salones de clase (universitarios, especialmente), y que ha recibido el nombre de Gamificación (Escaravajal y MartínAcosta, 2019; Lozada y Betancur, 2016; Marín et al., 2016; Pascuas et al., 2017).

De acuerdo con personas autoras de España, el uso de esta estrategia didáctica ha permitido la consolidación de los objetivos principales de las lecciones con aprendizajes más significativos, dinámicas de trabajo en equipo, motivación por las clases y el contenido en el alumnado (Etxeberria, 2008; Escaravajal y Martín-Acosta, 2019).

\section{Algunos inconvenientes de los juegos de video en educación}

La utilización de los videojuegos en el ámbito educativo no ha estado exenta de percepciones u opiniones en contra. Por lo general, los argumentos están basados en los supuestos efectos que tenían sobre el comportamiento de las personas, especialmente, las menores de edad. Algunos autores como Guerra (2015) y Ramón (2018), explican que la mayoría de las animadversiones generadas sobre los videojuegos fueron motivadas por las conjeturas realizadas por investigaciones y medios de comunicación que hacían eco continuamente de imprecisiones sobre resultados parciales mal interpretados, o bien a raíz de la emisión de criterios con base en prejuicios.

Se destacan en este aspecto varios resultados, principalmente, los expuestos por
Guerra (2015) y Martín del Pozo et al. (2017), quienes en sus pesquisas encontraron que en las investigaciones que exploran o miden los efectos de los videojuegos en entornos educativos formales - casi sin importar su procedencia-, no existe una metodología que siga un eje común de estandarización, lo cual dificulta que los resultados puedan ser contrastados o comparados con otros estudios similares.

Igualmente, se ha considerado que con excepción de algunos Serious Games hechos para propósitos muy puntuales, los juegos de video deben ser utilizados como un complemento para el trabajo en el aula, y que las investigaciones posean un proceso de evaluación o medición previo a los logros de aprendizaje y posterior a estos, por ejemplo (Lozada y Betancur, 2016; Pascuas et al., 2017).

Sin embargo, también se han señalado que existen escazas evidencias sobre los efectos de los videojuegos educativos, debido a que su valor como herramientas lúdicas o divertidas no basta para ser recursos didácticos efectivos necesariamente, más si su aplicación se centra únicamente en la repetición de movimientos o ejercicios, por encima a los procesos de comprensión como en el caso de aquellos enfocados en alfabetización o matemáticas, en fin, su utilidad podría cuestionarse. Una situación similar sucede con la gamificación, pues para algunas autoras $\mathrm{y}$ algunos autores, el uso de esta -centrada en actividades, puntuaciones, retos, etc.- puede llevar a que las pruebas de evaluación no se consideren como algo serio o no produzcan ningún aprendizaje (Etxeberria, 2011; Marín et al., 2016; 
Oceja y González, 2018; Ramón, 2018; Escaravajal y Martín-Acosta, 2019).

Finalmente, también se ha mencionado que el uso problemático de videojuegos comunes puede tener importantes repercusiones en el rendimiento escolar de las personas, principalmente, por los problemas comportamentales asociados a estos (Tejeiro et al., 2009; Moncada y Chacón, 2012; Lancheros et al., 2014; Soto, 2014; Guerra, 2015; Notiulcom, 2018).

\section{Videojuegos y violencia}

La mayoría de la literatura revisada para este trabajo hace alusión de este punto, tanto de forma directa como indirecta. En los apartados anteriores, se mencionaba cómo ciertas posturas o sesgos, con respecto a los juegos de video decantaron en prejuicios y asociaciones apresuradas de estos, como causantes primordiales de problemas tanto en la salud como en la educación. Al partir de los análisis realizados por personas autoras (Tejeiro et al., 2009; Etxeberria, 2011; Valcárcel, 2013; García, 2018), parece ser que la polémica ha estado desde el mismo origen comercial de estos en los años 60 y 70, engarzada especialmente, por coyunturas muy particulares o hasta endogámicas de los principales mercados de este tipo de entretenimiento, como en el caso de Estados Unidos, por ejemplo.

Ante esto, no es de extrañar que la violencia y los juegos de video con mecánicas de disparo o lucha sean uno de los temas de estudio más habituales directa e indirectamente en los espacios académicos de investigación o de abordaje de disciplinas de ciencias sociales, de la salud y de la educación en América Latina y España (Etxeberria, 2011; Soto, 2014; Guerra, 2015). Así, se ha mantenido como una vertiente de investigación constante el estudio de los efectos o la influencia que estos tienen sobre el comportamiento -agresivo- de poblaciones menores de edad o adolescente, en comparación con otras industrias del entretenimiento como la televisión, el cine, la literatura o los medios de comunicación como periódicos, telenoticieros, etc. (Gómez del Castillo, 2005; Etxeberria, 2008; Tejeiro et al., 2009; Etxeberria, 2011; Moncada y Chacón, 2012; Gray, 2014; García, 2018; Fuenmayor, 2018).

Fue en la década de los 90 cuando, ante las constantes presiones sociales y políticas, se inició la emisión de declaraciones que afirmaban tener mayor certidumbre sobre la relación entre los brotes o las acciones violentas registradas en países como Estados Unidos, el uso de juegos de video y sus efectos sobre el comportamiento de quienes jugaban con estos, a pesar de que no se contaba con resultados concluyentes que establecieran relaciones más allá de coocurrencias o asociaciones poco o nada significativas -en el caso de estudios cuantitativos- (Etxeberria, 2011; Fuenmayor, 2018; García, 2018).

Uno de los actores sociales más citados sobre este aspecto, quizás, sean los sectores conservadores y representantes de la Asociación Nacional del Rifle de Estados Unidos, los cuales especialmente después de incidentes de tiroteos masivos, responsabilizaban a los videojuegos como las inspiraciones directas para las personas que los perpetran (Gómez del Castillo, 
2005; Fuenmayor, 2018). Sin embargo, de acuerdo con una investigación realizada por Vossekuil, Fein, Reddy, Borum y Modzeleski (2004), para el Servicio Secreto y el Ministerio de Educación estadounidenses, solo aproximadamente un $12 \%$ de los tiradores en incidentes en escuelas preferían con regularidad.

En un talante similar, otras personas autoras han señalado que muchas de las investigaciones realizadas sobre estos temas: se sostienen sobre sesgos más que por argumentos científicos; tienden a mostrar resultados contradictorios; poco acuerdo o rigurosidad sobre los indicadores con los que se miden variables como la agresividad; el establecimiento de relaciones causales entre reacciones violentas y uso de videojuegos de disparos o de luchas con estudios correlacionales o asociativos, no explicativos o predictivos, etc. (Gómez del Castillo, 2005; Tejeiro et al., 2009; Valcárcel, 2013; Gray, 2014; García, 2018; Fuenmayor, 2018).

Asimismo investigaciones revisadas por parte de personas autoras de las autoras de Latinoamérica y España, han acotado que el uso de videojuegos violentos ha tenido relación con la disminución de actos de agresión, coincidiendo con las fechas de lanzamiento en unos casos y en la presencia de menores niveles de delitos violentos (Tejeiro et al., 2009; Fuenmayor, 2018).

\section{Desventajas asociadas al uso de juegos violentos}

Dicho lo anterior, puede hablarse en este aspecto de ciertos elementos que parecen estar asociados al uso de videojuegos violentos, sin olvidar que todas las relaciones señaladas no son causales.

En primera instancia, aunque se reconoce la necesidad de mayores investigaciones tanto en los contextos anglosajones como en América Latina y España, se ha mantenido el postulado de que el uso de videojuegos con contenidos violentos podría relacionarse con varios efectos comportamentales sobre aquellas personas que los utilizan, como: procesos cognitivos distorsionados de la conducta agresiva, aislamiento social y conducta delictiva o antisocial -al partir fundamentalmente, de registros anecdóticos-, etc. (Tejeiro et al., 2009; Etxeberria, 2011; Moncada y Chacón, 2012; Valcárcel, 2013; Gray, 2014; Ortiz-Huerta, et al., 2018; Parra-Moreno et al., 2018).

Por otra parte, se sostiene que el uso excesivo de juegos de video con contenidos violentos podría favorecer la falta de empatía, desensibilizar a quienes juegan ante la violencia, y suprimir conductas prosociales - aspecto que inclusive podría agravarse dependiendo del grupo étnico al que pertenezca la persona ${ }^{4}$ (Gómez del Castillo, 2005; Pindado, 2005; Etxeberria, 2011; de Oliveira et al., 2018).

También, se ha dejado claro por parte de algunas personas investigadoras que parece haber una fuerte relación entre la presencia de una personalidad agresiva y el uso abusivo de juegos de video violentos, pero no viceversa, contrario a las creencias popularizadas al respecto, ya que en

4 De acuerdo con algunos resultados referidos en las RSL, parecen ser las personas blancas "caucásicas" el grupo más propenso a reaccionar de forma violenta sobre asiáticos y latinoamericanos. 
el desarrollo de una personalidad de este tipo intervienen tanto factores ambientales, como una composición familiar determinada, la presencia de experiencias de abuso o maltrato, la falta de recursos económicos o sociales, entre otros (Moncada y Chacón, 2012; García, 2018). Eso sí, se ha expresado alerta por los tipos de contenido explícito de violencia presentes en algunos títulos, como un aspecto a tener en consideración (Tejeiro et al., 2009; García, 2018).

\section{Discusión de los resultados}

Los documentos de revisiones sistemáticas, metaanálisis y algunos reportes de prensa de estos, han sido un indicador importante para recoger una imagen a gran escala de qué ha ocupado a buena parte de la academia, tanto en Latinoamérica, España y un par de países de habla inglesa.

Más de la mitad de los trabajos se realizaron en los últimos cuatro años, mostrando por un lado que la producción de investigaciones relativas al videojuego ha venido aumentando, y por otro los temas e intereses de estas. De forma que los estudios se han dedicado a indagar a fondo las aplicaciones, ventajas y desventajas de estos en los campos de la salud y la educación. Entre tanto, varios trabajos exploraron las posibles relaciones de los estos programas con la violencia que parecían estos provocar. Así el recorrido parece moverse entre la exploración de sus potencialidades y los efectos de su uso en salud, educación y violencia.

En lo que respecta al campo de la salud, parece que las destrezas motrices y cognitivas que se desarrollan en quienes juegan con estos, se han convertido en objetivos e indicadores claves de logro en ámbitos hospitalarios de Latinoamérica, España y países de habla inglesa, especialmente, en procesos o tratamientos de rehabilitación de varias afecciones derivadas de accidentes o del deterioro de las facultades mentales perdidas por edad avanzada o enfermedades asociadas a esta.

Para el caso de la educación formal, los resultados analizados de países latinoamericanos y España sugieren que el uso de juegos de video puede incrementar la motivación por el trabajo y el proceso de enseñanza aprendizaje que se realiza en el aula. Especialmente, cuando el diseño de las actividades contempla aspectos determinantes como estar basados en el desarrollo de un contenido didáctico y aplicarse mediante dinámicas que involucren procesos o competencias grupales por encima de las individuales.

El tema de la violencia en los videojuegos y la que estos presuntamente ocasionan, ha llamado la atención en este trabajo por aparecer de forma indirecta en casi todos los documentos revisados, al tiempo que algunos de estos aparentan dar por un hecho su vinculación con comportamientos agresivos y episodios de ataques masivos a multitudes, anunciados con frecuencia en medios de comunicación. Sin embargo, se ha hecho un aporte sustancial al puntualizar - por parte de algunos autores- que un análisis adecuado de este tema debe incluir una mayor diversidad de variables, debido a que lo violento tiene lugar en los contextos sociales por causa de una multiplicidad de factores que no pueden ser reductibles a un tipo 
específico de estos programas interactivos o un título en particular.

Por otra parte, sobre las desventajas o inconvenientes del uso de juegos de video, se ha mantenido el interés por buscar o encontrar una relación causal entre el uso de estos y el desarrollo de adicciones o utilización problemática de ellos. Así como establecer una asociación entre la emergencia de una vida sedentaria, las complicaciones de salud derivadas, y problemas de rendimiento escolar. No obstante, un señalamiento frecuente de las personas autoras de las RSL es que en su mayoría las investigaciones revisadas presentan problemas en las operacionalizaciones de los objetos de estudio, en los postulados teóricos y metodológicos utilizados para su análisis, así como las conclusiones generadas.

De manera que parte de este problema radica en que las investigaciones primarias revisadas por las personas autoras con frecuencia excluyen muchas variables en el análisis cuantitativo o cualitativo que realizan. Usando en el caso de las primeras, metodologías e instrumentos estadísticos que llegan únicamente a estudios de alcance correlacional y con resultados de asociación poco o nada significativos para establecer inferencias con un grado de validez, confiabilidad o predictibilidad aceptable. Mientras que en lo que respecta a los estudios cualitativos se tiende a realizar procesos de generalización de casos particulares que no llegan a representar el grueso de los atributos de las poblaciones generales, con las que se intentan equiparar o representar, y tener así, resultados o conclusiones que no van más allá del caso particular del que se está investigando y poco o nada tiene que ver con la población o grupo de personas jugadoras de videojuegos.

Estas complicaciones de comprensión, diseño, ejecución y comunicación de resultados de investigación sobre videojuegos y los fenómenos alrededor de estos, han sido reportados mayormente, en los estudios que han indagado en los campos de la educación y la comprensión de la violencia. Precisamente, ámbitos en los que parecen estar más cercanos o presentes los estereotipos, prejuicios y desinformaciones sobre los videojuegos como se advirtió en los apartados anteriores.

\section{Conclusiones y posibles rumbos de investigación en los estudios del videojuego}

A lo largo de este estudio, a grandes rasgos sobre el estado de la investigación del videojuego, un recurso imprescindible ha sido partir de las revisiones sistemáticas y los metaanálisis realizados por las personas investigadoras consultadas. Inicialmente, porque con este tipo de trabajos fue posible conocer una visión global de las tendencias, las perspectivas y las disciplinas que han predominado en el estudio de este campo.

Consecuentemente, se ha podido observar que el videojuego como objeto de estudio ha tocado fibras científicas, profesionales, políticas y sociales, con niveles de profundidad o atención, quizás en grados solo alcanzados por pocos temas u objetos de análisis o discusión, cuya relevancia en las diferentes sociedades hacen posible tal resonancia. 
Junto con lo anterior se ha denotado la presencia o el surgimiento de una variedad tendencias de estudios que van desde aquellas posturas que defienden los beneficios del uso de videojuegos, tanto en el nivel individual como grupal, hasta aquellos posicionamientos que se erigen en función de adjudicarle a estos objetos o fenómenos socioculturales la responsabilidad de los "males" de la sociedad y las juventudes actuales.

De tal suerte que, como se ha citado anteriormente, es posible observar que el estudio del videojuego padece de ciertas dificultades que han venido aminorándose hasta el presente -año 2019-. Las cuales correspondían a una conjunción de detalles comprendidos entre la poca claridad en los abordajes teóricos basados en extrapolaciones de conceptos, criterios de mediciones $\mathrm{y}$ estimaciones que no fueron desarrolladas para la indagación de estos, sino para valorar: adicciones a sustancias no permitidas; trabajar en áreas específicas de la salud y la medicina; la evaluación de procesos de educación formal (áulico) en la que quienes se encuentran en los pupitres son meras unidades receptoras del aprendizaje o agentes parciales de este; el análisis de medios de comunicación tradicionales; entre otras aplicaciones. Así, es común encontrarse investigaciones con fundamentos poco rigurosos para el desarrollo de los propios estudios y con la característica conjunta de no poseer una definición clara o adecuada de qué es un programa interactivo de este tipo, o las dinámicas que suceden al usarlo o jugar con este.

En respuesta a lo anterior, varias personas investigadoras han intentado resolver estas situaciones enfocándose en elementos sobre el estudio del juego, provenientes de varias disciplinas como antropología, historia, psicología, ciencias políticas, artes visuales, comunicación social, sociología, informática, lógica, matemática, entre otras. Esto desde el postulado de que, si bien se trata de una aplicación informática, su objetivo es ser utilizado con fines lúdicos y de entretenimiento, evitando así caer en puntos de vista confusos de los primeros estudios. Parte de los desarrollos de estas corrientes académicas han tenido importante presencia en países latinoamericanos y en España en la última década.

Ahora bien, ante todo lo expuesto hasta ahora, ¿cuáles podrían ser las próximas tendencias o puntos de partida para los procesos de investigación? Precisamente, uno de los rumbos que podría tomar la investigación del video juego, debería evitar los errores cometidos por las personas profesionales en el pasado, particularmente, en aquellas indagaciones que analicen temas de estos relacionados con los campos de la educación y la violencia, espacios en los que, al menos en esta revisión, parecen estar presentes mayormente las falencias referidas ampliamente en este artículo, con algunas excepciones en el campo de la salud.

En este sentido, personas autoras como Mäyrä (2008), Planells (2013), López (2014), Rodríguez (2016) y Ramón (2018), son solo una parte de la gran variedad de autores latinoamericanos, españoles y angloparlantes que discuten en el nivel teórico-epistemológico sobre el concepto y las características o las propiedades de los videojuegos como objeto 
de estudio, marcando junto con otras personas profesionales y otros profesionales una tendencia de investigación que en la actualidad, se ha vuelto más que necesaria de cara a la producción de pesquisas que puedan conformar un campo de estudio que ayude tener una mayor claridad teórica que pueda dar respuestas a algunas de las interrogantes que hoy todavía se realizan en diversas latitudes.

Por otra parte, a juzgar por los campos en los que se decantaron las RSL y los metaanálisis encontrados en este trabajo, existe un espacio de investigación pendiente de abarcar, y ese es el análisis sociocultural del videojuego, ya que una exploración inicial del detalle del comportamiento de los estudios en esta rama parece un esfuerzo importante a desarrollar.

En un sentido similar, se abre la posibilidad de estudiar los procesos y las formas de producción de los juegos de video, eso sí abarcando espacios más allá de un análisis económico -es decir: inversión, gastos y ganancias-, que expongan las condiciones particulares o generales que permiten la consolidación de estos desde el momento de su creación o planeamiento.

De la mano con esto, se da la necesidad de fortalecer el desarrollo de investigaciones que analicen los contenidos, las discursos y los imaginarios plasmados en los juegos de video, sería un aporte importante en términos de conocer los sustratos políticos, filosóficos, geopolíticos o culturales que les dan forma, al tiempo que se aproveche este trabajo para comprender la manera en la que son recibidos por personas que los juegan.

\section{Referencias}

Batchelor, J. (2018, diciembre 18). Global games market value rising to $\$ 134.9 b n$ in 2018. Recuperado de Gamesindustry.biz website: https://www.gamesindustry.biz/ articles/2018-12-18-global-gamesmarket-value-rose-to-usd134-9bnin-2018

Beltrán-Carrillo, V., Valencia-Peris, A., y Molina-Alventosa, J. (2011). Los videojuegos activos y la salud de los jóvenes: Revisión de la investigación. Revista Internacional de Medicina y Ciencias de la Actividad Física y el Deporte, 10(41), 203-219.

de Oliveira, N., Mena, A. J., y de Lima, I. I. (2018). Efectos de los géneros de videojuegos en la cognición de adultos sanos: Una revisión sistemática. Revista Neuropsicología Latinoamericana, 10(3), 19-29. https://doi. org/10.5579/rnl.2018.455

Escaravajal, J. C. y Martín-Acosta, F. (2019). Análisis biliográfico de la gamificación en educación Física. Revista Iberoamericana de la actividad física y el deporte., 8(1), 97-109. http://dx.doi.org/10.24310/ riccafd.2019.v8i1.5770

Etxeberria, F. (2011). Videojuegos violentos y agresividad. Pedagogía Social. Revista Interuniversitaria, (18), 31-39.

Etxeberria, F. (2008). Videojuegos, consumo y educación. Revista Electrónica Teoría de la Educación. Educación y Cultura en la Sociedad de la Información, 9(3), 11-28. 
Friese, S. (2013). ATLAS. ti 7: User Guide and Reference. Berlin: ATLAS. ti Scientific Software Development $\mathrm{GmbH}$.

Fuenmayor, A. (2018). Videojuegos y violencia: ¿hay causalidad? [Periodismo]. Recuperado el 2 de abril de 2019, de El Definido website: https://www.eldefinido.cl/actuali$\mathrm{dad} / \mathrm{mundo} / 10514 /$ Videojuegos-yviolencia-hay-causalidad/

García, S. (2018). Videojuegos y violencia: Una revisión de la línea de investigación de los efectos. Revista de la Escuela de Ciencias de la Educación, 1(14), 149-165.

Gray, P. (2014). Las ventajas de jugar a videojuegos para los niños (A. Pardo Romero, Trad.). Recuperado el 1 de abril de 2019, de ZOLANI website: http://zolani.es/ventajas-de-losvideojuegos/\#comments

Guanipa, M. (2008). ¿Cómo hacer un ensayo científico? Revista Electrónica de Humanidades, Educación y Comunicación Social, 3(5), 1-10.

Guerra, J. (2015). Revisión bibliográfica del paradigma digital game based learning (Master). Universidad de Extremadura, Cáceres.

Hernández, J. F., Cano, Á. P., y Parra, M. C. (2015). Taxonomía del videojuego: Un planteamiento por géneros. Revista Latina de Comunicación Social, 90, 2149-2168.

Humanante-Ramos, P., García-Peñalvo, F. J., y Conde-González, M. (2017). Entornos personales de aprendizaje móvil: Una revisión sistemática de la literatura. Revista Iberoamericana de Educación a Distancia, 20(2), 73-92. http://dx.doi.org/10.5944/ ried.20.2.17692

Lancheros, M. J., Amaya, M. A., y Barquero, L. A. (2014). Videojuegos y adiccion en niños-adolescentes: Una revisión sistemática. Revista de Terapia Ocupacional Galicia, 20(11), 1-22.

López, I. (2014). ¿Qué es un videojuego? Claves para entender el mayor fenómeno cultural del siglo XXI (2014a ed.). España: Ediciones Arcade.

Lozada, C., y Betancur, S. (2016). La gamifcación en la educación superior: Una revisión sistemática. Revista Ingenierías Universidad de Medellin, 16(31), 97-124. https://doi. org/10.22395/rium.v16n31a5

Marín, H., Alor, G., Zatarain, R., y Barron, M. L. (2016). Una revisión sistemática de técnicas de gamification en aplicaciones educativas inteligentes. Research in Computing Science, 111, 149-163. https://pdfs semanticscholar.org/27cd/00be38022a379b894c6 0c29ac8c42b20fea5.pdf

Martín del Pozo, M., García-Valcarcel, A., y Basilotta, V. (2017). Participación educativa en el desarrollo de serious games sobre bullying y uso seguro de Internet: Caminando se hace el camino. Revista Interuniversitaria de Investigación en Tecnología Educativa, (3), 13-24. http://dx.doi. org/10.6018/riite/2017/312881

Mäyrä, F. (2008). An Introduction to Game Studies: Games in Culture (1 st edition). London: SAGE Publications. 
Mercola. (2017, agosto 3). Cómo afectan los videojuegos al cerebro. Recuperado el 3 de marzo de 2019, de mercola: Tóme el control de su salud website: https://articulos. mercola.com/sitios/articulos/archivo/2017/08/03/como-los-videojuegos-afectan-el-cerebro.aspx

Merino, C., y Cárcamo, H. (2016). Los beneficios de los videojuegos interactivos: Una aproximación educativa y una revisión sistemática de la actividad física. New Approaches in Educational Research, 5(2), 122-130. https://doi.org/10.7821/ naer.2016.7.164

Moncada, J., y Chacón, Y. (2012). El efecto de los videojuegos en variables sociales, psicológicas y fisiológicas en niños y adolescentes. Retos. Nuevas tendencias en Educación Física, Deporte y Recreación, 21, 43-49.

Notiulcom. (2018). Violento juego de videojuegos para niños vinculado a una mayor agresión. Recuperado de https://www.notiulti.com/violentojuego-de-videojuegos-para-ninosvinculado-a-una-mayor-agresion/

Oceja, J., y González, N. (2018). Videojuegos y aprendizaje ¿Por qué la gamificación y los juegos educativos no son suficientes? En Á. Torres-Toukoumidis y L. RomeroRodríguez (Eds.), Gamificación en Iberoamérica: Experiencias desde la comunicación y la educación (Primer edición). Quito, Ecuador: Editorial Universitaria Abya-Yala.
Ortiz-Huerta, H., Pérez-de-Heredia-Torres, M., Guijo-Blanco, V., y Santamaría-Vázquez, montserrat. (2018). Eficacia de la intervención con videoconsolas en pacientes con ictus: Revisión sistemática. Revista de Neurología, 66(2), 49-58. https:// doi.org/10.33588/rn.6602.2017405

Parra-Moreno, M., Rodríguez-Juan, J., y Ruiz-Cárdenas, J. (2018). Efectos de la terapia con videojuegos comerciales sobre el equilibrio postural en pacientes con esclerosis múltiple: Revisión sistemática y metaanálisis de ensayos clínicos controlados aleatorizados. Neurología, (En prensa), 1-7. https://doi. org/10.1016/j.nrl.2017.12.001

Pascuas, Y. S., Vargas, E. O., y Muñoz, J. I. (2017). Experiencias motivacionales gamifcadas: Una revisión sistemática de literatura. Innovación Educativa, 17(75), 63-80.

Pindado, J. (2005). Las posibilidades educativas de los videojuegos. Una revisión de los estudios más significativos. ixel-Bit. Revista de Medios y Educación, 26, 55-67.

Planells, A. J. (2013). La emergencia de los Game Studies como disciplina propia: Investigando el videojuego desde las metodologías de la Comunicación. Historia y Comunicación Social, 18, 519-528. http://dx.doi. org/10.5209/rev_HICS.2013. v18.43985

Ramón, L. S. (2018). Un mapa de los estudios latinoamericanos y españoles sobre videojuegos. Observatorio (OBS*) Journal, 12(1), 
147-168. https://doi.org/10.15847/ obsOBS12120181170

Rodríguez, Y. (2007). El ensayo académico: Algunos apuntes para su estudio. Sapiens. Revista Universitaria de Investigación, 8(1), 147-159.

Rodríguez, R. (2016). Videojuegos: La explosión digital que está cambiando al mundo. (Primera Edición). Sevi1la, España: Héroes de Papel.

Sánchez, M., Collado, S., Martín, P., y Cano de la Cuerda, R. (2018). Apps en Neurorehabilitación. Una revisión sistemática de aplicaciones móviles. 33(5), 313-326. https:// doi.org/10.1016/j.nrl.2015.10.005

Sánchez, V. (2009). Los videojuegos ganaron en ventas a los discos de DVD y Blu-ray en 2008. 20 minutos. Recuperado de https://www.20minutos. es/videojuegos/noticia/dvd-blurayvideojuego-446090/0/\#xtor=AD$15 \mathrm{yxts}=467263$

Segalás, A. (2011, marzo 3). El uso de videojuegos retrasa el deterioro cognitivo en EA. Diario Marca. Recuperado de https://cuidateplus.marca. com/enfermedades/2011/03/03/videojuegos-retrasa-deterioro-cognitivo-ea-22440.html

Soto, R. (2014). Descripción del estado actual de la adicción a los videojuegos a partir de una revisión bibliográfica integrativa (Licenciatura, Universidad de Chile). Recuperado de http://repositorio.uchile.cl/ handle/2250/135632

Stanmore, E., Stubbs, B., Vancampfort, D., de Bruin, E., y Firth, J. (2017). The effect of active video games on cognitive functioning in clinical and non-clinical populations: A meta-analysis of randomized controlled trials. Neuroscience and Biobehavioral Reviews, 78, 34-43. http://dx.doi.org/10.1016/j. neubiorev.2017.04.011

Tejeiro, R., Pelegrina del Río, M., y Gómez, J. L. (2009). Efectos psicosociales de los videojuegos. Comunicación, 7(1), 235-250. Recuperado de 2 marzo 2019.

Toril, P. (2015). Envejecimiento cognitivo: Efectos del entrenamiento con videojuegos en la memoria de trabajo viso-espacial de mayores sanos (universidad Nacional de Educacion a Distancia). Recuperado de http://e-spacio.uned.es/fez/view/ tesisuned:Psicologia-Ptoril

Uchuypoma, D. (2017). Juegos online: Una mirada desde el juego patológico. Hamut'ay, 4(2), 55-64. http://dx.doi.org/10.21503/hamu. v4i2.1472

Valcárcel, C. (2013). El videojuego como recurso didáctico en el aprendizaje de las matemáticas en primer curso de educación secundaria (Trabajo Fin de Master). Recuperado de https://reunir.unir.net/bitstream/ handle/123456789/2134/Valcarcel_TFM\%20\%28entregado\%20 por $\% 201$ a $\% 20$ Directora $\% 29$. pdf? sequence $=1 \mathrm{yis}$ Allowed $=\mathrm{y}$

Vela, J. A., Velázquez, J., y Veyna, M. (2017). Videojuegos basados en BCI (Interface cerebro computadora): Revisión Sistemática Literaria. Programación Matemática y Software, 
9(2). Recuperado de https://www. progmat.uaem.mx:8080/Vo19num2/ vo19num2art2.pdf

Vossekuil, B., Fein, R., Reddy, M., Borum, R., y Modzeleski, W. (2004). The final report and findings of the safe school initiative: Implications for the prevention of school attacks in the United States (p. ingles) [Final Inform]. Recuperado de United States Secret Service and United States Departmet of education website: https://www2.ed.gov/admins/lead/ safety/preventingattacksreport.pdf
Wijman, T. (2018, abril 30). Mobile Revenues Account for More Than 50\% of the Global Games Market as It Reaches \$137.9 Billion in 2018. Recuperado de Newzoo website: https://newzoo.com/insights/articles/global-games-market-reaches137-9-billion-in-2018-mobilegames-take-half/

Wolf, M., y Perron, B. (2005). Introducción a la teoría del videojuego. Formats Revista de Comunicación Audiovisual, 4. Recuperado de https:// www.raco.cat/index.php/Formats/ article/download/257329/344420 